\title{
Derivations Determined by Multipliers on Ideals of a $C^{*}$-Algebra ${ }^{1)}$
}

By

\author{
George A. ElliotT*
}

\begin{abstract}
Sakai's theorem that every derivation of a simple $C^{*}$-algebra is determined by a multiplier is generalized, in the class of separable approximately finite-dimensional $C^{*}$-algebras, as follows. It is shown that, in such a $C^{*}$-algebra, any derivation can be approximated arbitrarily closely in norm by a derivation which is determined by a multiplier on a nonzero closed two-sided ideal. It is shown, moreover, that the multiplier may be chosen to have norm bounded by fixed multiple of the norm of the derivation.
\end{abstract}

\section{1.}

Examples constructed in [1] and in [6] show that, if a $C^{*}$-algebra does not have a minimal closed two-sided ideal, it may have a derivation the restriction of which to no nonzero closed two-sided ideal is determined by a multiplier. Nevertheless, it seems reasonable to expect that the set of such derivations has empty interior. The purpose of this paper is to verify this in a class of $C^{*}$-algebras which lends itself particularly to technical analysis.

Theorem. Let $A$ be the $C^{*}$-algebra inductive limit of a sequence of finite-dimensional $C^{*}$-algebras, and let $D$ be $a$ derivation of $A$. Then for each $\varepsilon>0$ there exist a nonzero closed two-sided ideal $I_{\varepsilon}$ of $A$, a multiplier $x_{\varepsilon}$ of $I_{\varepsilon}$ such that $\left\|x_{\varepsilon}\right\| \leqq 248\|D\|$, and a derivation $D_{\varepsilon}$ of $A$ such that $\left\|D-D_{\varepsilon}\right\| \leqq \varepsilon$ and $D_{\varepsilon}\left|I_{\varepsilon}=\operatorname{ad} x_{\varepsilon}\right| I_{\varepsilon}$.

Communicated by H. Araki, July 18, 1974. Revised July 31, 1974.

* Mathematics Institute, University of Copenhagen, Denmark.

1) This work was done while the author was a visiting member of the Research Jnstitute for Mathematical Sciences, Kyoto University. 
The theorem is a consequence of lemmas 2.2 and 3.6 below.

2.

First we shall show that, roughly, a derivation when sufficiently reduced vanishes asymptotically.

2.1. Lemma. Let $B_{1}, \ldots, B_{n}$ be simple finite-dimensional $C^{*}$-algebras, and set $B_{1} \otimes \cdots \otimes B_{n}=B$. Let $D$ be a derivation of $B$ such that $D B_{1} \subset$ $B_{1}, \ldots, D B_{n} \subset B_{n}$. Then

$$
\left\|D\left|B_{1}\|+\cdots+\| D\right| B_{n}\right\| \leqq 2\|D\| .
$$

Proof. Write $D=D_{1}+i D_{2}$ where $D_{1}$ and $D_{2}$ are skew-adjoint-preserving derivations of $B$. Then the proof of 6 of [2] shows that

$$
\left\|D_{j}\left|B_{1}\|+\cdots+\| D_{j}\right| B_{n}\right\| \leqq\left\|D_{j}\right\|, \quad j=1,2 .
$$

The conclusion follows from the inequalities

$$
\|D\| \leqq\left\|D_{1}\right\|+\left\|D_{2}\right\| \leqq 2\|D\|
$$

2.2. Lemma. Let $A$ be the $C^{*}$-algebra inductive limit of a sequence of finite-dimensional $C^{*}$-algebras, let $D$ be a derivation of $A$, and let $\varepsilon>0$. Then there exists a nonzero simple finite-dimensional sub-C*. algebra $B$ of $A$ such that, if $e$ denotes the unit of $B, P_{e}$ the map $A \ni$ $a \mapsto e a e \in e A$, and $B^{\prime}$ the commutant of $B$ in $A$,

$$
\left\|P_{e} D \mid e B^{\prime}\right\| \leqq \varepsilon
$$

Proof. By hypothesis there exists an increasing sequence $A_{1} \subset A_{2} \subset \cdots$ of finite-dimensional sub- $C^{*}$-algebras of $A$ with union dense in $A$. It is enough to prove the lemma for $D$ belonging to a dense set of derivations. Therefore, by 2.3 of [3] we may suppose that $D \cup A_{k} \subset \cup A_{k}$.

Suppose that the conclusion of the lemma is false. We shall deduce an inequality in contradiction with 2.1 .

Choose $n=3,4, \ldots$ such that $n^{-1} 2\|D\| \leqq \varepsilon$, and set $n^{-1} 2\|D\|=\delta$. Choose $k_{1}=1,2, \ldots$ such that $\left\|D \mid A_{k_{1}}\right\|>\delta$. Choose $k_{2}>k_{1}$ such that $D A_{k_{1}} \subset A_{k_{2}}$, and choose a minimal central projection $e_{2}$ in $A_{k_{2}}$ such that 


$$
\left\|P_{e_{2}} D \mid A_{k_{1}}\right\|>\delta
$$

Choose $k_{3}>k_{2}$ such that $D A_{k_{2}} \subset A_{k_{3}}$ and choose $k_{4}>k_{3}$ such that $D A_{k_{3}} \subset A_{k_{4}}$.

Note that $\left\|P_{e_{2}} D \mid e_{2} A_{k_{4}}^{\prime}\right\|>\delta$; otherwise, with $f$ a minimal central projection in $e_{2} A_{k_{4}} e_{2}$ is simple, we would have $f\left(f A_{k_{4}} f\right)^{\prime} \subset e_{2} A_{k_{4}}^{\prime}$ and so $\left\|P_{f} D \mid f\left(f A_{k_{4}} f\right)^{\prime}\right\| \leqq \delta \leqq \varepsilon$, which was assumed not to hold.

The algebra $\cup e_{2} A_{k} e_{2} \cap e_{2} A_{k_{4}}^{\prime}$ is dense in $e_{2} A_{k_{4}}^{\prime}$; therefore there exists $k_{5}>k_{4}$ such that $\left\|P_{e_{2}} D \mid e_{2} A_{k_{5}} e_{2} \cap e_{2} A_{k_{4}}^{\prime}\right\|>\delta$. Choose $k_{6}>k_{5}$ such that $D A_{k_{5}} \subset A_{k_{6}}$. Since $D A_{k_{3}} \subset A_{k_{4}}$, by 7 of [2] $D\left(A_{k_{4}}^{\prime}\right) \subset A_{k_{3}}^{\prime}$. Since $e_{2} \in A_{k_{4}}$ and $e_{2} D\left(e_{2}\right) e_{2}=0$ (see proof of 3.4 below), $P_{e_{2}} D\left(e_{2} A_{k_{4}}^{\prime}\right)=e_{2} D\left(e_{2}\right) A_{k_{4}}^{\prime} e_{2}+$ $e_{2} D\left(A_{k_{4}}^{\prime}\right) e_{2}=e_{2} D\left(A_{k_{4}}^{\prime}\right) e_{2} \subset e_{2} A_{k_{3}}^{\prime}$. This shows that

$$
P_{e_{2}} D\left(e_{2} A_{k_{5}} e_{2} \cap e_{2} A_{k_{4}}^{\prime}\right) \subset e_{2} A_{k_{6}} e_{2} \cap e_{2} A_{k_{3}}^{\prime} .
$$

Choose a minimal central projection $e_{6}$ in $e_{2} A_{k_{6}} e_{2} \cap e_{2} A_{k_{3}}^{\prime}$ such that

$$
\left\|P_{e_{0}} D \mid e_{2} A_{k_{5}} e_{2} \cap e_{2} A_{k_{4}}^{\prime}\right\|>\delta \text {. }
$$

Choose $k_{7}>k_{6}$ and $k_{8}>k_{7}$ such that $D A_{k_{6}} \subset A_{k_{7}}$ and $D A_{k_{7}} \subset A_{k_{8}}$. As above, $\left\|P_{e_{6}} D \mid e_{6} A_{k_{8}}^{\prime}\right\|>\delta$, and there exists $k_{9}>k_{8}$ such that $\| P_{e_{6}} D \mid$ $e_{6} A_{k_{9}} e_{6} \cap e_{6} A_{k_{8}}^{\prime} \|>\delta$. Choose $k_{10}>k_{9}$ such that $D A_{k_{9}} \subset A_{k_{10}}$. Then, as above,

$$
P_{e_{6}} D\left(e_{6} A_{k_{9}} e_{6} \cap e_{6} A_{k_{8}}^{\prime}\right) \subset e_{6} A_{k_{10}} e_{6} \cap e_{6} A_{k_{7}}^{\prime},
$$

and so we may choose a minimal central projection $e_{10}$ in $e_{6} A_{k_{10}} e_{6} \cap$ $e_{6} A_{k_{7}}^{\prime}$ such that

$$
\left\|P_{e_{10}} D \mid e_{6} A_{k_{9}} e_{6} \cap e_{6} A_{k_{8}}^{\prime}\right\|>\delta
$$

Continue this process until $n$ projections $e_{2} \geqq \cdots \geqq e_{4 n-2}$ have been chosen, and denote $e_{4 n-2}$ by $e$. The algebras

$$
e_{2} A_{k_{2}}, e_{6}\left(e_{2} A_{k_{6}} e_{2} \cap e_{2} A_{k_{3}}^{\prime}\right), e_{10}\left(e_{6} A_{k_{10}} e_{6} \cap e_{6} A_{k_{7}}^{\prime}\right), \ldots
$$

are pairwise commuting simple finite-dimensional sub- $C^{*}$-algebras of $A$, and so also are the algebras

$$
e A_{k_{2}}, e\left(e_{2} A_{k_{6}} e_{2} \cap e_{2} A_{k_{3}}^{\prime}\right), e\left(e_{6} A_{k_{10}} e_{6} \cap e_{6} A_{k_{7}}^{\prime}\right), \ldots
$$


The latter algebras have a common unit $e$; denote them by $B_{1}, \ldots, B_{n}$ and denote the algebra they generate by $B$.

Since $B \subset e A e, P_{e} D$ is a derivation from $B$ into $e A e$. We shall now show that $P_{e} D B_{i}$ commutes with $B_{j}$ for distinct $i, j=1, \ldots, n$. By 7 of [2] it is enough to consider the case $i<j$, which is clear from the relations

$$
\begin{aligned}
& B_{2} \subset e^{\prime} \cap\left(e_{2} A_{k_{3}} e_{2}\right)^{\prime} \subset\left(e A_{k_{3}} e\right)^{\prime}, B_{3} \subset e^{\prime} \cap\left(e_{6} A_{k_{1}} e_{6}\right)^{\prime} \subset\left(e A_{k_{1}}\right)^{\prime}, \ldots, \\
& P_{e} D B_{1}=P_{e} D\left(e A_{k_{2}}\right)=e D\left(A_{k_{2}}\right) e \subset e A_{k_{3}} e, \\
& P_{e} D B_{2}=P_{e} D\left(e\left(e_{2} A_{k_{6}} e_{2} \cap e_{2} A_{k_{3}}^{\prime}\right)\right)=e D\left(e_{2} A_{k_{6}} e_{2} \cap e_{2} A_{k_{3}}^{\prime}\right) e \subset e A_{k_{7}} e_{,} \ldots .
\end{aligned}
$$

From $\left\|P_{e_{2}} D \mid A_{k_{1}}\right\|>\delta$, simplicity of $e_{2} A_{k_{2}}$, and $e \in e_{2} A_{k_{2}}^{\prime}$ follows $\| P_{e} D \mid$ $A_{k_{1}} \|>\delta$, whence $\left\|P_{e} D \mid e A_{k_{1}}\right\|>\delta$. From $\left\|P_{e_{6}} D \mid e_{2} A_{k_{5}} e_{2} \cap e_{2} A_{k_{4}}^{\prime}\right\|>\delta$, simplicity of $e_{6}\left(e_{2} A_{k_{6}} e_{2} \cap e_{2} A_{k_{3}}^{\prime}\right)$ and $e \in e_{6}\left(e_{2} A_{k_{6}} e_{2} \cap e_{2} A_{k_{3}}^{\prime}\right)^{\prime}$ follows $\| P_{e} D \mid e_{2} A_{k_{5}} e_{2}$ $\cap e_{2} A_{k_{4}}^{\prime} \|>\delta$, whence $\left\|P_{e} D \mid e\left(e_{2} A_{k_{5}} e_{2} \cap e_{2} A_{k_{4}}^{\prime}\right)\right\|>\delta$. It is possible to continue in this way.

Let $P$ be a projection of norm one from $e A e$ onto $B$; then (see e.g. 2 of [2]) $\bar{D}=P P_{e} D$ is a derivation of $B$ such that $\bar{D} B_{1} \subset B_{1}, \ldots, \bar{D} B_{n} \subset$ $B_{n}$. Since $P_{e} D\left(e A_{k_{1}}\right) \subset B_{1}, P_{e} D\left(e\left(e_{2} A_{k_{5}} e_{2} \cap e_{2} A_{k_{4}}^{\prime}\right)\right) \subset B_{2}, \ldots$, the preceding paragraph shows that $\left\|\bar{D}\left|B_{1}\|>\delta, \ldots,\| \bar{D}\right| B_{n}\right\|>\delta$. Hence by 2.1,

$$
2\|D\|=n \delta<\left\|\bar{D}\left|B_{1}\|+\cdots+\| \bar{D}\right| B_{n}\right\| \leqq 2\|\bar{D}\| \leqq 2\|D\| \text {. }
$$

This contradiction completes the proof of the lemma.

\section{3.}

We shall now use relations between derivations of an algebra and of a reduced subalgebra, established in [3], to complement the preceding result.

3.1. Lemma. Let $A$ be the $C^{*}$-algebra inductive limit of a sequence of finite-dimensional $C^{*}$-algebras, let $e$ be a projection in $A$, and let $D$ be a derivation of eAe. Then there exists a derivation $D_{0}$ of $A$ such that $\left\|D_{0}\right\| \leqq 3\|D\|$ and $D_{0} \mid e A e=D$.

Proof. This is the statement of 4.5 of [3], except for the estimate 
of the norm of the extension, which can be obtained by examining the proof of 4.5 of [3].

3.2. Lemma. Let $A$ be the $C^{*}$-algebra inductive limit of a sequence of finite-dimensional $C^{*}$-algebras, let $D$ be a derivation of $A$, and let $e$ be a projection in $A$. Suppose that $D \mid e A e=0$. Denote by $I$ the closed two-sided ideal of $A$ generated by $e$. Then there exists a multiplier $z$ of $I$ such that $\|z\| \leqq 16\|D\|$ and $D|I=\operatorname{ad} z| I$.

Proof. This is the statement of 4.4 of [3], except for the estimate of the norm of the multiplier, which can be obtained by examining the construction described in 4.4 of [3].

3.3. Lemma. Let $A$ be the $C^{*}$-algebra inductive limit of a sequence of finite-dimensional $C^{*}$-algebras, let $D$ be a derivation of $A$, and let $e$ be a projection in $A$. Suppose that $D(e A e) \subset e A e$ and that $\|D \mid e A e\| \leqq$ $\varepsilon$. Denote by $I$ the closed two-sided ideal of $A$ generated by $e$. Then there exist a derivation $D_{1}$ of $A$ and a multiplier $z$ of $I$ such that $\left\|D-D_{1}\right\| \leqq 3 \varepsilon,\|=\| \leqq 16\|D\|+48 \varepsilon$, and $D_{1}|I=\operatorname{ad} z| I$.

Proof. By 3.1 there exists a derivation $D_{0}$ of $A$ such that $\left\|D_{0}\right\| \leqq$ $3\|D \mid e A e\| \leqq 3 \varepsilon$ and $D_{0}|e A e=D| e A e$. Set $D-D_{0}=D_{1}$. Then $\left\|D-D_{1}\right\|=$ $\left\|D_{0}\right\| \leqq 3 \varepsilon$, and $D_{1} \mid e A e=0$. Hence by 3.2 there exists a multiplier $z$ of $I$ such that

$$
\|z\| \leqq 16\left\|D_{1}\right\| \leqq 16\|D\|+16\left\|D_{0}\right\| \leqq 16\|D\|+48 \varepsilon
$$

and $D_{1}|I=\operatorname{ad} z| I$.

3.4. Lemma. Let $A$ be the $C^{*}$-algebra inductive limit of a sequence of finite-dimensional $C^{*}$ algebras, let $D$ be a derivation of $A$, and let $e$ be a projection in $A$. Suppose that $\left\|P_{e} D \mid e A e\right\| \leqq \varepsilon$, where $P_{e}$ denotes the map $A \in a \mapsto e a e \in e A$. Denote by I the closed two-sided ideal of $A$ generated by $e$. Then there exist a derivation $D_{1}$ of $A$ and a multiplier $w$ of $I$ such that $\left\|D-D_{1}\right\| \leqq 3 \varepsilon,\|w\| \leqq 82\|D\|+48 \varepsilon$, and $D_{1} \mid I$ $=\operatorname{ad} w \mid I$.

Proof. Since $e^{2}=e$, we have $D(e) e+e D(e)=D(e), 2 e D(e) e=e D(e) e$, 
$e D(e) e=0$; hence $[[D(e), e], e]=D(e)$. Set $\quad D-\operatorname{ad}[D(e), e]=D_{2}$. Then $D_{2}(e A e) \subset e A e$, so $D_{2}\left|e A e=P_{e} D_{2}\right| e A e$. Again since $e D(e) e=0, P_{e}$ ad $[D(e)$, $e] \mid e A e=0$. Hence $D_{2}\left|e A e=P_{e} D_{2}\right| e A e=P_{e} D\left|e A e ;\left\|D_{2} \mid e A e\right\| \leqq \varepsilon\right.$.

By 3.3 there exist a derivation $D_{3}$ of $A$ and a multiplier $z$ of $I$ such that $\left\|D_{2}-D_{3}\right\| \leqq 3 \varepsilon,\|z\| \leqq 16\left\|D_{2}\right\|+48 \varepsilon \leqq 80\|D\|+48 \varepsilon$, and $D_{3} \mid I=$ ad $z \mid I$.

Set $\quad D_{3}+\operatorname{ad}[D(e), e]=D_{1} \quad$ and $\quad z+[D(e), e]=w$. Then $\left\|D-D_{1}\right\|=$ $\left\|D_{2}-D_{3}\right\| \leqq 3 \varepsilon, w$ is a multiplier of $I$ with $\|w\| \leqq\|z\|+\|[D(e), e]\| \leqq 82\|D\|+$ $48 \varepsilon$, and $D_{1}|I=\operatorname{ad} w| I$.

3.5. Lemma. Let $A$ be a $C^{*}$-algebra with unit, let $D$ be a derivation of $A$, and let $B$ be a simple finite-dimensional sub-C*algebra of $A$ with the same unit as $A$. Suppose that $\left\|D \mid B^{\prime}\right\| \leqq \varepsilon$, where $B^{\prime}$ denotes the commutant of $B$ in $A$. Then there exists $y \in A$ such that $\|y\| \leqq\|D\|$ and $\| D-$ ad $y \| \leqq 3 \varepsilon$.

Proof. Let $U$ be a finite subgroup of the unitary group of $B$ generating $B$ as a linear space. Following [5], set $n^{-1} \sum_{u \in U} D(u) u^{*}=y$, where $n$ is the number of elements of $U$. Then $D|B=\operatorname{ad} y| B$. (If $v \in U$ then $v y v^{*}+D(v) v^{*}=n^{-1} \sum_{u \in U} v D(u) u^{*} v^{*}+D(v) v^{*}=n^{-1} \sum_{u \in U}\left(D(v u) u^{*} v^{*}-D(v) u u^{*} v^{*}\right)$ $+D(v) v^{*}=y ; D(v)=[y, v]$.) Moreover, $\|y\| \leqq\|D\|$.

If $b \in B^{\prime}$ then for each $u \in U$,

$$
[D(u), b]=D(u b)-u D(b)-D(b u)+D(b) u=[D(b), u] ;
$$

since

$$
[y, b]=n^{-1} \sum_{u \in U}\left[D(u) u^{*}, b\right]=n^{-1} \sum_{u \in U}[D(u), b] u^{*}
$$

we have

$$
\|[y, b]\|=\left\|n^{-1} \sum_{u \in U}[D(b), u] u^{*}\right\| \leqq 2\|D(b)\| \leqq 2 \varepsilon\|b\| .
$$

This shows that $\left\|(D-\operatorname{ad} y) \mid B^{\prime}\right\| \leqq \varepsilon+2 \varepsilon=3 \varepsilon$. Since $(D-\operatorname{ad} y) \mid B=0$, by the proof of 4.1 of [3] we have $\|D-\operatorname{ad} y\| \leqq 3 \varepsilon$.

3.6. Lemma. Let $A$ be the $C^{*}$-algebra inductive limit of a sequence of finite-dimensional $C^{*}$-algebras, let $D$ be a derivation of $A$, and let 
$B$ be a simple finite-dimensional sub-C*-algebra of $A$, with unit $e$. Suppose that

$$
\left\|P_{e} D \mid e B^{\prime}\right\| \leqq \varepsilon,
$$

where $P_{e}$ denotes the map $A \ni a \rightarrow$ i $\rightarrow e \in e A e$ and $B^{\prime}$ denotes the commutant of $B$ in $A$. Denote by $I$ the closed two-sided ideal of $A$ generated by $e$. Then there exist a derivation $D_{1}$ of $A$ and a multiplier $x$ of $I$ such that $\left\|D-D_{1}\right\| \leqq 9 \varepsilon,\|x\| \leqq 247\|D\|+144 \varepsilon$, and $D_{1}|I=\operatorname{ad} x| I$.

Proof. By 3.5 there exists $y \in e A e$ such that $\|y\| \leqq\left\|P_{e} D\right\| \leqq\|D\|$ and $\left\|\left(P_{e} D-\operatorname{ad} y\right) \mid e A e\right\| \leqq 3 \varepsilon$. Then $\left\|P_{e}(D-\operatorname{ad} y) \mid e A e\right\| \leqq 3 \varepsilon$, whence by 3.4 there exist a derivation $D_{2}$ of $A$ and a multiplier $w$ of $I$ such that $\| D-\operatorname{ad} y-$ $D_{2}\|\leqq 9 \varepsilon\| w,\|\leqq 82\| D-\operatorname{ad} y\|+144 \varepsilon \leqq 264\| D \|+144 \varepsilon$, and $D_{2}|I=\operatorname{ad} w| I$. Set $D_{2}+\operatorname{ad} y=D_{1}, y+w=x$. Then $\left\|D-D_{1}\right\| \leqq 9 \varepsilon,\|x\| \leqq\|y\|+\|w\| \leqq 247\|D\|+$ $144 \varepsilon$, and $D_{1}\left|I=\left(D_{2}+\operatorname{ad} y\right)\right| I=(\operatorname{ad} w+\operatorname{ad} y)|I=\operatorname{ad} x| I$.

\section{Questions and Remarks}

4.1. Examination of the proof of 1 shows that $I_{\varepsilon}$ may be chosen so that $I_{\varepsilon} \not \subset J$, where $J$ is a given proper closed two-sided ideal of $A$. It is not at all clear though whether $I_{\varepsilon}$ can be chosen to be essential, i.e., with zero annihilator.

A related question is whether $D_{\varepsilon}$ and $I_{\varepsilon}$ may be chosen so that also the image of $D_{\varepsilon}$ in $A / I_{\varepsilon}$ is determined by a multiplier. A weaker requirement is that $D_{\varepsilon}$ may be chosen so that for some composition series $\left(I_{\alpha}\right)$ of $A$ the derivation of each $I_{\alpha+1} / I_{\alpha}$ induced by $D_{\varepsilon}$ is determined by a multiplier.

4.2. A modification of the techniques of this paper, incorporating the methods of [4], shows that if an automorphism of a separable approximately finite-dimensional $C^{*}$-algebra leaves closed two-sided ideals invariant and in each irreducible representation is extendible to the weak closure, then it is approximable arbitrarily closely in norm by an automorphism determined by a multiplier on a nonzero closed two-sided ideal, which may be chosen not to lie in a given proper closed twosided ideal. 
It follows that such an automorphism is extendible in any representation to an inner automorphism of the weak closure. (One constructs a composition series in each quotient of which the automorphism is close in norm to an automorphism determined by a multiplier, and applies the theorems of Kadison and Ringrose that an automorphism close in norm to the identity is the exponential of a derivation, and of Sakai and Kadison that a derivation is extendible in any representation to an inner derivation of the weak closure.) This generalizes the implication (iii) $\Rightarrow$ (ii) of Theorem 3.2 of [4], to the class of $C^{*}$-algebras considered, i.e., inductive limits of sequences of finite-dimensional $C^{*}$-algebras.

\section{References}

[1] Akemann, C. A., Elliott, G. A., Pedersen, G. K. and Tomiyama, J., Derivations and multipliers of $C^{*}$-algebras, Amer. J. Math., to appear.

[2] Elliott, G. A., Derivations of matroid $C^{*}$-algebras, Invent. Nath. 9 (1970), 253267.

[3] Elliott, G. A., On lifting and extending derivations of approximately finitedimensional $C^{*}$-algebras, J. Functional Analysis 17 (1974), 395-408.

[4] Lance, E. C., Inner automorphisms of UHF algebras, J. London Math. Soc. 43 (1968), 681-688.

[5] Johnson, B. E. and Ringrose, J. R., Derivations of operator algebras and discrete group algebras, Bull. London Math. Soc. 1 (1969), 70-74.

[6] Tomiyama, J., Derivations of $C^{*}$-algebras which are not determined by multipliers in any quotient algebra, Proc. Amer. Math. Soc. 47 (1975), 265-267. 